\title{
Cost-utility analysis of a nationwide vaccination programme against serogroup B meningococcal disease in Israel
}

\author{
Gary M. Ginsberg $\cdot$ Colin Block $\cdot$ Chen Stein-Zamir
}

Received: 18 November 2015/Revised: 31 March 2016/Accepted: 4 April 2016/Published online: 22 April 2016

(C) Swiss School of Public Health (SSPH+) 2016

\begin{abstract}
Objectives Using cost-utility analysis, to evaluate whether or not to adopt a Neisseria meningitidis serogroup B vaccination programme for Israeli children.

Methods Epidemiological, demographic, health service utilisation and economic data were integrated into a spreadsheet model to calculate the cost per averted disability-adjusted life year (DALY) of the intervention.

Results Assuming $78 \%$ vaccine efficacy with no herd immunity, vaccination will prevent 223 cases and 22 deaths over a 100-year period. Based on vaccine price of $\$ 60$ per dose, total intervention costs $(\$ 315,400,000)$ are partially offset by a $\$ 22,700,000$ reduction in treatment and sequelae costs as a result of decreased morbidity. The
\end{abstract}

Electronic supplementary material The online version of this article (doi:10.1007/s00038-016-0821-0) contains supplementary material, which is available to authorized users.

\section{G. M. Ginsberg $(\bowtie)$}

Department of Technology Assessment, Public Health Service,

Ministry of Health, Jerusalem, Israel

e-mail: gary.ginsberg@moh.health.gov.il;

gmginsberg@gmail.com

\section{Block}

Department of Clinical Microbiology and Infectious Diseases, Hadassah-Hebrew University Medical Centre, Ein Kerem, Jerusalem, Israel

\section{Stein-Zamir}

Jerusalem District Health Office, Ministry of Health, Jerusalem, Israel

\section{Stein-Zamir}

Braun School of Public Health and Community Medicine, The Hebrew University and Hadassah, Ein Kerem, Jerusalem, Israel intervention was not cost-effective since the net cost $(\$ 292,700,000)$ per averted DALY gained (1249 mostly due to decreased mortality) was $\$ 234,394$. Additional two dose catch-up programmes vaccinating children in cohorts aged 1-2 to 1-13 were also not cost-effective.

Conclusions The vaccination will become cost-effective if vaccine costs fall below $\$ 19.44$ per dose. However, in identified high risk areas, the vaccine would be cost-effective and could be recommended for use both with and without catch-up campaigns.

Keywords Cost-utility analysis - Meningococcal B . Vaccination · Economic evaluation

\section{Introduction}

Invasive meningococcal disease (IMD) accounts worldwide for around 75,000 deaths annually (Lozano et al. 2012). Incidence rates correlate with age and socio-economic deprivation especially crowded housing.

Although disease incidence is at historic lows, the overall case-fatality ratio (CFR) ranges between 5 and $10 \%$ in developed countries (Healy et al. 2002; Pouwels et al. 2013; Ben-Shimol et al. 2012; Scholten et al. 1994). Some of these survivors acquire long-term sequelae (e.g., neurologic disability, limb or digit loss, and hearing loss).

The introduction of vaccinations against other meningococcal serotypes ( $\mathrm{A}, \mathrm{C}, \mathrm{W}, \mathrm{Y})$ has caused a shift towards serogroup $\mathrm{B}$ becoming the main cause of IMD in high income countries (Sridhar et al. 2015), though in Israel it has been dominant since reliable records became available in the mid-1970s.

Australasia (especially New Zealand), Europe (especially Eire and Eastern Norway), North America 
(especially Eastern Canada), and South America (especially Chile) had high incidence of MEN B. In contrast, China, India and sub-Saharan Africa (except for South Africa) had very few cases (Sridhar et al. 2015). The CFR from serogroup B meningococcal disease (MEN B) ranges between 3 and $10 \%$ in non-outbreak scenarios (Sridhar et al. 2015). There can also be substantial morbidity due to long-term sequelae in survivors (Centres for Disease Control and Prevention 2005), despite the fact that sequelae-related damage has decreased, due to medical care improvements, including earlier diagnostic recognition.

In Israel, overall IMD incidence rates declined from 1.7 to $1.0 / 100,000$ during the past 30 years (Israel Center for Disease Control 2012). Meningitis constituted $69.2 \%$ of all cases, the most common serogroup being MENB which accounted for around three-quarters of IMD case isolates (76.9\%) (Ben-Shimol et al. 2012).

Prevention and control of IMD is a public health challenge due to the rapid disease onset, occasional fulminant course, and subsequent sequelae and mortality. Efficacious vaccines against serogroups A, C, Y, W135 are available. A licensed (Gorringe and Pajon 2012), immunogenic and tolerable MENB vaccine (Gill 2013) received positive endorsement from the European Medicines Agency (Novartis 2012) (Bexsero, Novartis) and approval from the US FDA (FDA News Release 2014). In September 2015, MEN $\mathrm{B}$ was added to the routine childhood immunization programme schedule in the UK (Hakin and Cosford 2015) after repeated cost-utility analyses (Wise 2014).

This study uses cost-utility analysis, to evaluate the possibility of instituting a nationwide vaccination programme of infants against MENB in Israel in terms of its cost per disability-adjusted life year (DALY) averted. The model could easily be adapted for use in other developed or developing countries.

\section{Methods}

\section{Data sources}

Data on cases in Israel during 2000-2010 (Erickson and De Wals 1998) were supplemented with data from 2011 to 2013 obtained from the Ministry of Health's Epidemiology department. Detailed information was obtained on 236 IMD cases ( $73 \%$ being MEN B) in the Jerusalem district from published data (Stein-Zamir et al. 2008, 2014), hospital files and laboratory results. Data from Jerusalem were extrapolated to the whole of Israel, whenever national data were not available. From these reports, we calculated national age-specific incidence rates, mortality and morbidity rates and case fatality rates for MEN B infections.
Cost-utility analysis: basic model

A Microsoft Excel spreadsheet model was constructed, incorporating vaccine efficacy, epidemiological, health service utilisation, demographic and economic data (listed with sources in Supplementary Material Appendix I). The model calculated the effect of vaccinations against MEN B (for a 10-year period) on incidence and mortality over a 100-year time horizon. Age, gender and ethnic population and birth cohort projections were based on the trend for the years 2006-2013 (Central Bureau of Statistics 2015).

The cost-utility ratio (CUR) calculated the net costs per averted disability-adjusted life year (DALY) added as a result of using the new vaccine, using the formula:

$$
\begin{aligned}
& \text { Net costs per averted DALY } \\
& =\frac{\text { Costs of vaccination }- \text { Savings in treating IMD }}{\text { DALYs averted from decreased mortality and morbidity }} .
\end{aligned}
$$

Costs are viewed from a societal perspective at mid2013 price levels at the average annual exchange rate of 3.61 shekels to the US dollar (Central Bureau of Statistics 2015). These included costs not only incurred by the health and welfare services but also of work absences and transport costs to receive treatment. All future costs and DALY were discounted at an annual rate of $3 \%$. Data on out-of-pocket expenses or DALYs averted from reduced caregiver burden were not available.

Vaccine efficacy

Unfortunately, at the time of constructing the model, there was a lack of published information on clonal complexes, protein variants and peptide identities which precluded us from making an Israeli-specific estimate of cases caused by strains covered by the vaccine. In the absence of Israelispecific strain data, we conservatively assumed the strain coverage of the vaccine to be the lower bound of a UK estimate of $66 \%$ (JCVI 2014; Tu et al. 2014) increasing to $88 \%$ in a sensitivity analysis (JCVI 2014), which takes into account the evidence that use of the meningococcal antigen typing system (MATS) assay, underestimated 4CMenB vaccine strain coverage (Frosi et al. 2013). Following recent recommendations for the UK (JCVI 2014), we assumed a three dose vaccine schedule (2, 4, 12 months) as opposed to a four dose schedule (Vesikari et al. 2013).

Vaccine efficacy against covered strains was assumed to be $90 \%$ (Pouwels et al. 2013; JCVI 2014; Tu et al. 2014, Christensen et al. 2013). We conservatively assumed the first dose offered no protection, but the second dose provided $47 \%$ overall protective efficacy (Arnold et al. 2011). Because of evidence of waning efficacy found in similar 
outer membrane vaccine types (Holst et al. 2003) we incorporated differential waning rates by age of vaccination $(<1,1-3$ and $>3$ years old) into the model [Personal Communication, Hules, Novartis] following a negative exponential trend similar to that found with MEN C vaccination (De Wals et al. 2011).

\section{Intervention costs}

We assumed a baseline vaccine price of $\$ 60$ per dose (range \$10-\$110), in keeping with vaccine prices used in previous cost-utility analyses (Pouwels et al. 2013; Tu et al. 2014; Christensen et al. 2013). Since at each point of the vaccine schedule the infant received other concurrent vaccinations, additional transport costs and work losses for bringing the child to the clinic were not included. Provision was made for treatment costs, transport costs and work losses arising from the visits to health service providers for vaccine side effects, mainly fever related (Vesikari et al. 2013; Gossger et al. 2012; McNicholas et al. 2007).

Incidence of GP visits for side effects of $0.23 \%$ was based on the $0.13 \%$ excess of fevers in persons (Vesikari et al. 2013) receiving MENB plus routine vaccinations $(0.74 \%)$ over persons receiving just routine vaccinations $(0.61 \%)$. Incidence of hospital visits of $0.00034 \%$ was obtained by adjusting GP visit incidence by the reported ratio of hospital to GP visit from a post-marketing trial in New Zealand (McNicholas et al. 2007). A sensitivity analysis was based on the 2.2 times higher vaccine related serious adverse events reported in another study (Gossger et al. 2012).

Vaccine wastage based on operational experience in Israel was around $0.5 \%$. Costs were included relating to nurse's and administration time, health education, syringes and swabs. Due to excess capacity in the system, no extra cold chain costs were incurred. Costs of vaccinating older children in nurseries or schools by public health nurses were calculated separately, with appropriate provision for travelling time and costs.

\section{Herd immunity}

Our baseline scenario assumed there was no herd immunity (see discussion). However, herd immunity was incorporated into a sensitivity analysis by assuming that the agespecific force of infection was directly proportional to the number of incident cases, treating the Jewish and nonJewish populations as separate sectors (i.e.: vaccinating a 1 -year old would only affect 1-year olds and not susceptible older children or adults). The reduced force of infection after vaccination was then applied to the exposed population (i.e.: unvaccinated persons who had not previously contracted MEN B).
This sectorial separation generally holds for persons in kinder-garden, school and army but is weaker when applied for university and workplace population interactions. A second sensitivity analysis on herd immunity assumed the effect of reduced force of infection applied across all age groups.

Costs of acute cases

Acute care costs were calculated by multiplying the expected number of cases by the unit costs of health services received. Chemoprophylaxis and follow-up costs, of contacts which included time taken to trace and visit contacts (Wright et al. 2013), were added.

\section{Chronic sequelae}

Incidence, costs and disability weight (DW) associated with chronic sequelae from MEN B are described in detail in the Supplementary Material (Appendix III).

\section{Averted DALY losses}

Total DALY losses averted were calculated by summing the morbidity and mortality DALY losses averted from decreased incidence of MENB as a result of vaccination (see Supplementary Material Appendix IV).

\section{Decision rules}

Taking into account the resources available in Israel, an intervention was defined as being very cost-effective and cost-effective if the cost per averted DALY is less than the per capita GNP of $\$ 36,167$ in 2013 (Central Bureau of Statistics 2015) or between 1 and 3 times the per capita GDP (\$36,167-\$108,501), respectively. If the cost per averted DALY is more than three times the GDP per capita $(\$ 108,501)$ then the intervention was regarded as not being cost-effective (WHO Commission on MacroEconomics and Health 2001).

\section{Sensitivity analyses}

One-way sensitivity analyses were carried out by varying the values of major input parameters (vaccination costs, incidence rates, waning rates, incidence and costs of sequelae, herd immunity, discount rate) in turn by $+20 \%$. This enabled elasticity's of the CUR (defined as \% change in CUR/\% change in major variable) to be compared across a homogeneous metric.

Two-way sensitivity analyses were carried out on the ex-ante expected major drivers of vaccination costs and carriage strain coverage. 
Three-way sensitivity analyses were carried out on by vaccination costs, incidence rates, carriage strain coverage along with adding two dose (JCVI 2014) catch-up campaigns for ranges of children aged $1-2, \ldots, 1-5$ (i.e. up to pre-school) ... 1-12 (i.e. up to senior school), 1-13.

\section{Results}

\section{Epidemiology}

During 2000-2013, 940 reported cases of IMD occurred in Israel (annual incidence rate 0.94/100,000). Around 699 cases $(74.4 \%)$ were caused by MEN B (annual incidence rate $0.70 / 100,000), 57.2 \%$ in males. The majority of MEN B cases $(85.7 \%)$ occurred in children $<15$ years, infants accounting for $36 \%$ of all cases (annual incidence rate of 12.0/100,000). Around 73 people died from MEN B $(\mathrm{CFR}=5.4 \%)$. The CFR was $4.5 \%$ in infants, and 6.7, 3.9 and $6.6 \%$ in persons aged $1-4,5-14$ and $15+$, respectively.

Cost-utility analysis, treatment costs

Applying the age-gender and ethnic-specific incidence rates from 2000 to 2013 to the 2013 population of $7,978,067$ (Central Bureau of Statistics 2015) gives an estimate of 56.1 cases of MEN B in 2013. Around $62.9 \%$ of these cases would require a visit to a primary care physician (PCP costing \$13.80).

Around $7.9 \%$ of MEN B deaths (0.24 cases) occur before the patient reaches the emergency room (ER) costing \$341 per case (Ginsberg et al. 2015). A further 0.24 cases die in the ER, costing $\$ 565$ per case (Ministry of Health 2012; Jacobs and Noseworthy 1990). Another 2.6 cases die in intensive care units (ICU). Around 55.3 cases are hospitalised (average cost of \$9511), based on 3.5 days in an ICU (Jacobs and Noseworthy 1990; Scott et al. 2002) and 6.1 days in a non-ICU department (Ministry of Health 2012). All 53 survivors utilise audio-visual screening tests (\$1007) and a post-discharge out-patient visit (\$77) (Ministry of Health 2012).

Chemoprophylaxis costs for the average of 41 contacts per case, of whom, approximately $5 \%$ are untraceable with $80 \%$ receiving post-exposure prophylaxis at a health centre, amount to $\$ 27.71$ per contact.

Vaccination results in their being 155 fewer cases, of which $95.5 \%$ were prevented during the first 25 years of the programme (Table 1). Average cost per incident case was $\$ 11,286$. Total projected costs of treating incident cases in the no vaccine scenario are $\$ 51.9$ million, falling to $\$ 49.1$ million if vaccinations were implemented (Table 2).
Table 1 Effects (2013-2112) of vaccinating Israeli cohort aged 0-1 for 10 years with/without $1-4$ years catch-up

\begin{tabular}{llll}
\hline & No vaccine & Vaccine & Difference \\
\hline Without catch-up & & & \\
Cases (2013-2028) & 1061 & 912 & 148 \\
Cases & 24,807 & 24,653 & 155 \\
Mortality & 1970 & 1954 & 16 \\
Potential years of life lost & 166,358 & 165,022 & 1336 \\
With catch-up & & & \\
Cases (2013-2028) & 1133 & 799 & 334 \\
Cases & 25,890 & 25,537 & 353 \\
Mortality & 2014 & 1978 & 37 \\
Potential years of life lost & 171,444 & 168,555 & 2888 \\
\hline
\end{tabular}

Sequelae incidence and costs (Table 2)

The neurological sequelae rate was $19.3 \%$ (Stein-Zamir et al. 2014). Discounted average lifetime costs (age-specific in the model, but presented for illustration just for the age group 1-4 years) of treating a case with severe neurological disorders [1.75\% of survivors (Stein-Zamir et al. 2014)] were $\$ 701,000$, mild-to-moderate neurological disorders [7.0\% (Stein-Zamir et al. 2014), \$256,000], light neurological disorders [10.5\% (Stein-Zamir et al. 2014), $\$ 29,400$ ], bilateral hearing loss $(1.75 \%, \$ 167,000)$, unilateral hearing loss $(1.75 \%, \$ 96,000)$, mild hearing disorder (3.5\%, \$20,700), seizures [1.75\% (Holst et al. 2003), $\$ 53,400$ (Scott et al. 2002)], scarring [3.9\% (Healy et al. 2002; Scholten et al. 1994; Edwards et al. 1981; Vesikari et al. 2013), $\$ 6900$ (Bettinger et al. 2013)], renal problems (3.5\% (Healy et al. 2002; Scholten et al. 1994; Erickson and De Wals 1998; Bettinger et al. 2013), \$121,000 (Erickson and De Wals 1998; Slack et al. 2005; Buysse et al. 2008, 2010)], digital amputations (0.64 \%, \$2954).

The total projected costs of treating sequelae in the novaccination scenario are $\$ 189,000,000$, almost four times the $\$ 49,000,000$ costs of treating the acute phase of MEN B (including chemoprophylaxis and follow-up). Costs of treating sequelae fall to $\$ 47,300,000$ in the vaccination scenario (Table 2).

Intervention costs

Persons receiving MENB plus routine vaccinations were assumed to have an excess $0.23 \%$ visits to a PCP (Vesikari et al. 2013) for medical attention (costing $\$ 13.80$ per visit) mainly on account of fevers, compared with recipients of routine vaccinations only. Excess hospitalizations in $0.00034 \%$ of vaccinations (Gossger et al. 2012) were assumed for an average of four days, costing $\$ 2244$ per admission. 
Table 2 Discounted costs, savings and costs per averted disability-adjusted life year (Israel 2013)

\begin{tabular}{|c|c|c|c|}
\hline & Without vaccine & Vaccine & Cost/savings \\
\hline Vaccination & $\$ 0$ & $\$ 315,438,622$ & $\$ 315,438,622$ \\
\hline Premature burial & $\$ 2,737,947$ & $\$ 2,636,357$ & $-\$ 101,591$ \\
\hline Incident cases & $\$ 49,380,739$ & $\$ 47,965,132$ & $-\$ 1,415,607$ \\
\hline Bilateral deafness & $\$ 11,674,086$ & $\$ 11,310,937$ & $-\$ 363,149$ \\
\hline Unilateral deafness & $\$ 6,708,586$ & $\$ 6,499,204$ & $-\$ 209,382$ \\
\hline Mild hearing disorder & $\$ 2,934,141$ & $\$ 2,843,405$ & $-\$ 90,736$ \\
\hline Severe neurological dis. & $\$ 58,237,103$ & $\$ 49,188,685$ & $-\$ 9,048,418$ \\
\hline Mild-moderate neurological disability & $\$ 73,884,169$ & $\$ 71,561,844$ & $-\$ 2,322,324$ \\
\hline Light neurological dis. & $\$ 11,966,081$ & $\$ 11,569,596$ & $-\$ 396,485$ \\
\hline Seizures & $\$ 3,928,347$ & $\$ 3,805,231$ & $-\$ 123,115$ \\
\hline Amputations & $\$ 144,697$ & $\$ 140,630$ & $-\$ 4,067$ \\
\hline Skin scarring & $\$ 1,178,281$ & $\$ 1,144,503$ & $-\$ 33,778$ \\
\hline Renal disease & $\$ 18,489,915$ & $\$ 17,955,724$ & $-\$ 534,191$ \\
\hline Total treatment costs & $\$ 241,264,093$ & $\$ 226,621,250$ & $-\$ 14,642,843$ \\
\hline Work losses incident & $\$ 8,094,832$ & $\$ 2,609,868$ & $-\$ 5,484,964$ \\
\hline Work losses sequelae & $\$ 191,482$ & $\$ 186,199$ & $-\$ 5,282$ \\
\hline Work losses severe sequelae & $\$ 123,233$ & $\$ 119,871$ & $-\$ 3,362$ \\
\hline Total work losses & $\$ 8,409,547$ & $\$ 2,915,938$ & $-\$ 5,493,609$ \\
\hline Total net intervention cost & $\$ 249,673,639$ & $\$ 544,975,811$ & $\$ 295,302,171$ \\
\hline Discounted DALYS added & & & 976 \\
\hline Cost per averted DALY & & & $\$ 302,629$ \\
\hline
\end{tabular}

The target population are the 170,819 (Central Bureau of Statistics 2015) infants born in 2013 and the subsequent nine years. While vaccine coverage would be nationwide (i.e. $100 \%$ ), compliance rates were assumed to be 93 and $98 \%$ in the Jewish and non-Jewish populations, respectively, based on coverage achieved for DTaP and Hepatitis B vaccinations (Central Bureau of Statistics 2015).

Total vaccination costs for a MEN B vaccination programme in 2013 amount to $\$ 32,500,000$ million, with (discounted) intervention costs over 10 years being $\$ 315,400,000$ (Table 2).

\section{Cost-utility ratios}

The 10-year vaccination programme would result in 155 fewer MEN B cases, 16 fewer deaths and an extra 1336 potential years of life added (Table 1). Total treatment costs of MEN B cases and sequelae would fall by $\$ 1.4$ and $\$ 13.1$ million, respectively. A further $\$ 102,000$ would be saved in delayed burial costs. In addition, around $\$ 5.5$ million would be saved due to decreased work absences, resulting in a total saving of $\$ 20.1$ million, resulting in a net programme cost of $\$ 295.3$ million (Table 2).

Decreased mortality, incident and sequelae cases would avert 964, 3.8 and 8.2 discounted DALYs, respectively (Table 3). The net cost ( $\$ 295.3$ million) per averted DALY (976) amounts to $\$ 302,630$ (Table 2), as this is in excess of thrice the per capita GDP threshold of $\$ 108,501$, the intervention is not cost-effective. If the vaccine strain coverage was $88 \%$ then the cost per averted DALY falls to $\$ 245,472$, which is still not cost-effective.

Catch-up campaigns

Costs ranged from a not cost-effective $\$ 302,590$ to $\$ 412,255$ per averted DALY if children aged 1-2 and 1-12, respectively, are included in the campaign (Fig. 1). Vaccine costs would have to fall below $\$ 19.33$ or $\$ 12.62$ per dose to ensure that the average cost per averted DALY of including children aged 1-5 (pre-schoolers) or aged 1-12 (pre senior-schoolers) in a national infant vaccination campaign would be cost-effective. For vaccine strain coverage of $88 \%$, cost per averted DALY ranged from $\$ 244,781$ to $\$ 332,617$ (Fig. 1). Vaccine costs would have to fall below $\$ 24.88$ or $\$ 16.86$ per dose, respectively, to attain cost-effectiveness for 1-2 and 1-12-year-old catchup campaigns.

Sensitivity analyses (Fig. 2 and Supplementary Material Appendix II)

Adjustment of the vaccine price by $20 \%$ to $\$ 72$ and $\$ 48$ per dose resulted in costs per averted DALY of $\$ 360,000$ and $\$ 245,000$, respectively. The break-even vaccine price 
Table 3 Disability-adjusted life years lost due to mortality and morbidity by intervention (Israel 2013)

\begin{tabular}{|c|c|c|c|c|c|c|}
\hline & \multirow[t]{2}{*}{ No vaccine } & \multirow[t]{2}{*}{ Vaccine } & \multirow[t]{2}{*}{ Difference } & \multicolumn{2}{|l|}{ Discounted } & \multirow[t]{2}{*}{ Difference } \\
\hline & & & & No vaccine & Vaccine & \\
\hline Mortality & 145,245 & 144,072 & 1173 & 26,630 & 25,666 & 964 \\
\hline Incident cases & 747 & 742 & 4.7 & 131 & 128 & 3.8 \\
\hline Bilateral deafness & 22.5 & 22.4 & 0.1 & 3.9 & 3.8 & 0.1 \\
\hline Unilateral deafness & 14.6 & 14.5 & 0.1 & 2.6 & 2.5 & 0.1 \\
\hline Mild hearing disorder & 22.3 & 22.1 & 0.1 & 3.9 & 3.8 & 0.1 \\
\hline Severe neurological dis. & 139.1 & 138.2 & 0.9 & 27.8 & 23.5 & 4.3 \\
\hline Mild-mod neurological dis. & 278.3 & 276.4 & 1.9 & 48.5 & 47.0 & 1.5 \\
\hline Light neurological dis. & 125.2 & 124.4 & 0.8 & 21.8 & 21.2 & 0.7 \\
\hline Seizures & 16.5 & 16.4 & 0.1 & 2.9 & 2.8 & 0.1 \\
\hline Amputations & 36.3 & 36.0 & 0.2 & 6.4 & 6.2 & 0.2 \\
\hline Skin scarring & 0.0 & 0.0 & 0.0 & 0.0 & 0.0 & 0.0 \\
\hline Renal disease & 71.1 & 70.7 & 0.5 & 13.0 & 12.2 & 0.8 \\
\hline Total & 146,717 & 145,535 & 1182 & 26,892 & 25,916 & 976 \\
\hline
\end{tabular}

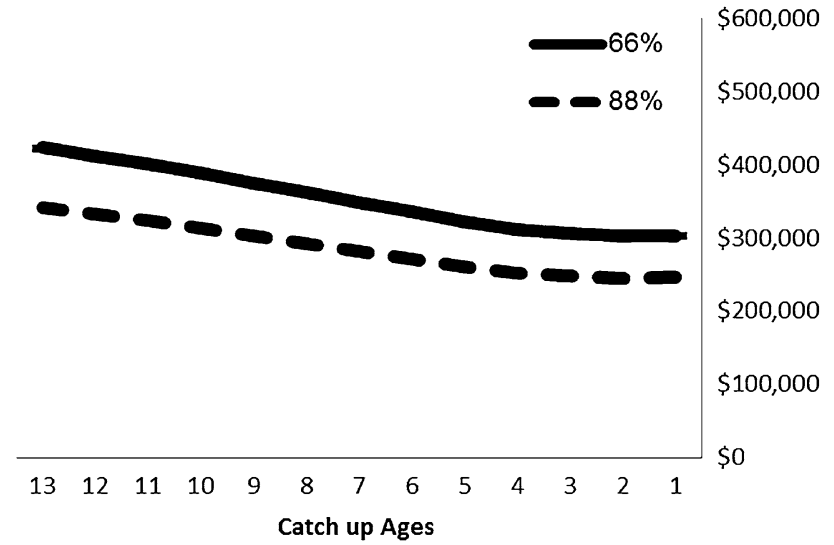

Fig. 1 Cost per disability-adjusted life year by catch-up ages and strain coverage (Israel 2003)

for cost-effectiveness is $\$ 19.44$ and rises to $\$ 24.88$ if the vaccine strain coverage rises to $88 \%$.

Because of the small size of the Jerusalem sequelae study, we carried out a sensitivity analysis of MEN B sequelae rates based on data from five $(n=1480)$ literature studies (Healy et al. 2002; Scholten et al. 1994; Erickson and De Wals 1998; Bettinger et al. 2013; Viner et al. 2012), except for mild-medium and light neurological sequelae where no alternative data (to our Jerusalem data) were available. An average DW of 0.19 with a cost of $\$ 57,710$ per amputee was imputed for amputations based on applying limb specific costs data (World Health Organization 1996) to the (sometimes multiple) limb specific distribution of amputee cases from the literature (Erickson and De Wals 1998; Slack et al. 2005; Viner et al. 2012; Buysse et al. 2009). After making
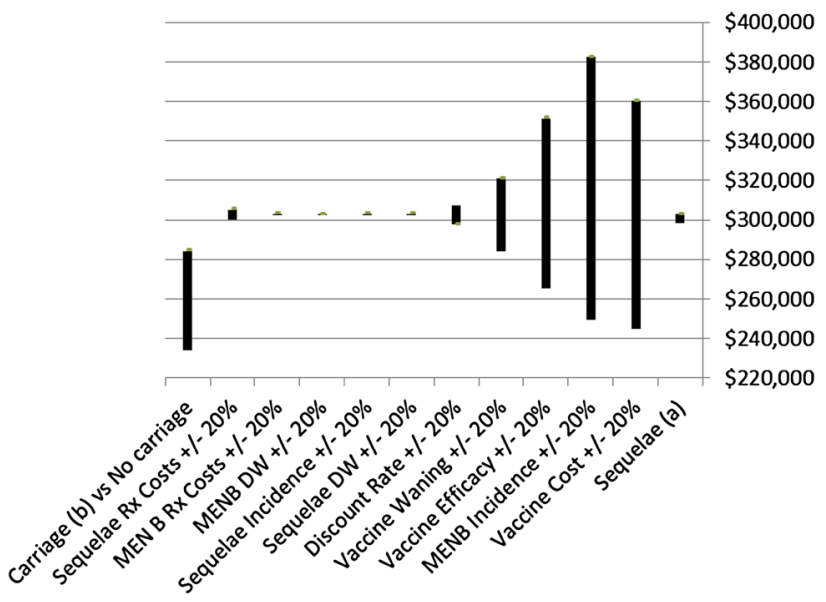

Fig. 2 Cost per disability-adjusted life year (one-way sensitivity analyses, Israel 2013)

all these adjustments the cost per averted DALY to $\$ 298,000$.

The CUR was sensitive to changes in the MEN B incidence rate, vaccine efficacy and its waning rate. However, to attain cost-effectiveness, incidence rates would have to rise from 0.70 to 1.77 per by 100,000 . Costeffectiveness would not be obtained $(\mathrm{CUR}=\$ 204,474)$ even with $100 \%$ vaccine efficacy against all strains (c.f. $66 \%)$ or had a $0 \%$ waning rate $(\mathrm{CUR}=\$ 199,563)$. The CUR was insensitive to changes in the discount rate, MEN $\mathrm{B}$, vaccine side effects, disability weights of sequelae or unit treatment costs (Fig. 2).

Assuming our baseline conservative assumption, that the new protein-based vaccine does not induce herd immunity is erroneous (Dellicour and Greenwood 2007), results in 

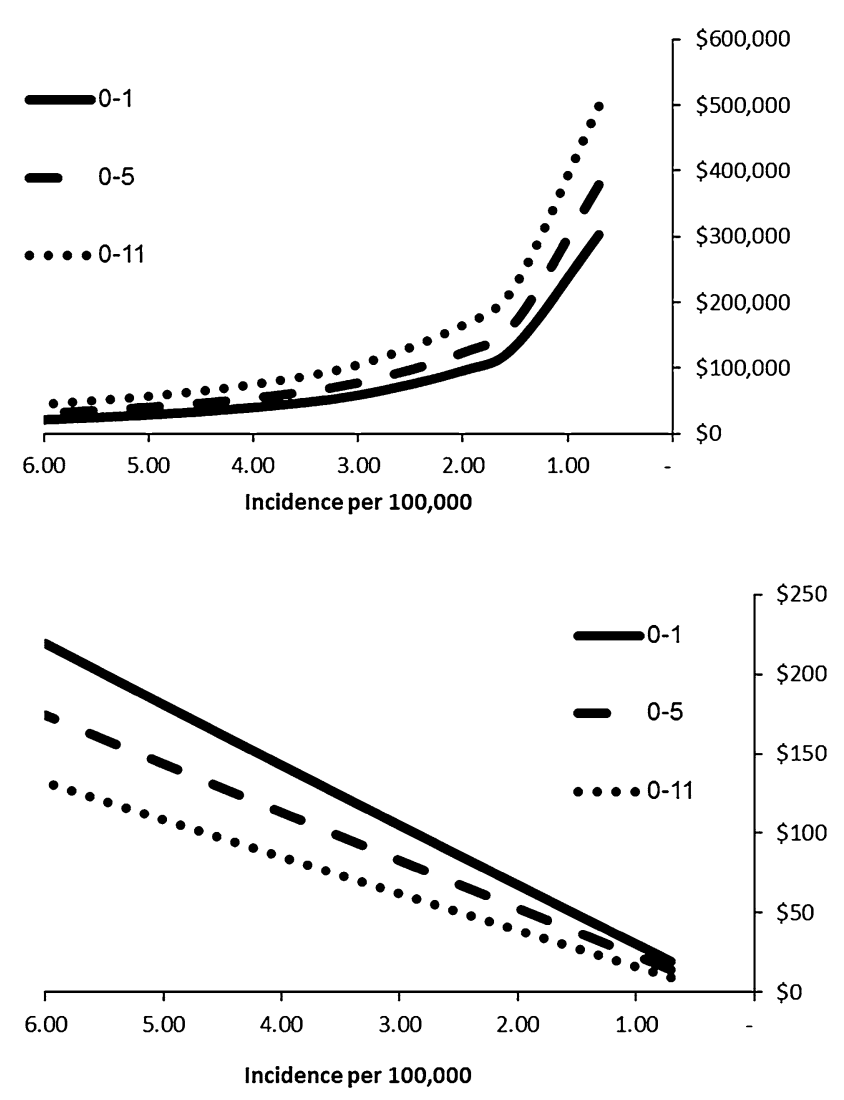

Fig. 3 Cost per disability-adjusted life year (upper) and break-even vaccine cost (lower) by incidence and age of vaccination (Israel 2013)

the model showing the cost per averted DALY decreasing to a still not cost-effective range of between $\$ 234,000$ and $\$ 284,000$ depending on whether carriage was within or across age groups (Fig. 2).

At the baseline price of $\$ 60$ per vaccine shot, threshold incidence rates for cost-effectiveness would be 1.83, 2.5 and 2.93 for catch-up campaigns covering infants, $0-5$ and 0-12-year olds, respectively (Fig. 3, upper). For illustration, assuming we could identify an area with $3 / 100,000$ incidence rates (about four times the national average), then the cost-effective vaccine price thresholds would be $\$ 105$ (around current price), $\$ 83$ and $\$ 62$, respectively (Fig. 3, lower).

\section{Discussion}

MENB is the leading cause of invasive meningococcal disease (IMD) in Israel. The availability of a new vaccine against MEN B prompted an ex-ante evaluation of a three dose childhood vaccination programme.

The price of the vaccine is obviously a major driver of costs per DALY averted. At $\$ 60$ per dose, the intervention cost of $\$ 302,630$ per DALY averted, clearly renders it not cost-effective. Vaccine prices would have to fall below $\$ 19.44$ and $\$ 4.32$ in order to render the intervention to be cost-effective or very cost-effective. Catch-up campaigns (based on a two dose schedule) would not be a cost-effective addition due to the lower child age-specific incidence rates. However, they could become cost-effective if the vaccine costs were to fall substantially. Focussed campaigns in high risk areas (such as universities) or at times of disease outbreaks are likely to be cost-effective even at present vaccine prices depending on the level of excess disease incidence.

Our vaccine price (used to attain the British $\$ 48,000$ cost-effectiveness threshold) threshold of $\$ 6.79$ was far smaller than the reported $\$ 24$ threshold vaccine price in a British modelling study (Christensen et al. 2013). This difference was primarily attributable to the British model being based on far higher disease incidence rates. In addition, the British model assumed herd immunity, which caused a $41 \%$ reduction in the cost-utility ratio (CUR) compared to their sensitivity analysis omitting herd immunity. A recent re-evaluation study suggested that routine infant vaccination could be cost-effective if the vaccine could be procured at $<20 \%$ of the list price of around $\$ 110$ a dose, although the cost-effective threshold vaccine price varied with different assumptions used in the modelling (Christensen et al. 2014).

A manufacturer-funded model developed by Novartis (Huels et al. 2013) incorporated all the elements of the burden of disease model (McNicholas et al. 2007) that favoured the adoption of the vaccine, resulting in a $\$ 12$ $\$ 33$ price range for economically justifiable infant vaccinations (compared to our models \$6.79) based on the same $\$ 48,000$ threshold. The most questionable of these assumptions being the accounting of lost productivity associated with a potential slight decrease in IQ for a significant proportion of survivors (Meningococcal B Pilot Project Task Group 2014).

In contrast, our costs per DALY averted were lower than those of a recent Dutch study (with no herd immunity) (Pouwels et al. 2013), despite incidence rates being about $50 \%$ higher than in Israel. The Dutch study reported the vaccine price would have to fall below $\$ 4.55$ (compared to $\$ 10.14$ in our study) in order for cost-effectiveness ratio to fall below their $\$ 64,000$ threshold. We can only speculate that, besides inevitable difference in model structures, our use of a three as opposed to a four dose schedule and higher vaccine efficacy (81.4 vs. $75 \%$ ) contributed to this difference.

Our reported cost per averted DALY was also far lower than the \$4.6 million reported from Ontario, Canada ( $\mathrm{Tu}$ et al. 2014). This was mainly attributable to the far lower annual incidence rates in Ontario (0.19 vs. 0.70/100,000) but also due to the Canadian study using a higher baseline 
vaccine price ( $\$ 72.83$ vs $\$ 60$ per dose), lower sequelae costs and being carried out just from a payer perspective and therefore not including benefits from decreased work losses.

A recent model (excluding herd immunity) based in Italy (Tirani et al. 2015), which has very low incidence of meningococcal disease, showed that at a vaccine price of $\$ 89$ per dose the introduction of routine infant Men B vaccinations is unlikely to be cost-effective.

A model based in Chile reported a $\$ 15$ per dose vaccine cost-effectiveness threshold, based on a single dose mass vaccination protocol that was used in an epidemic outbreak scenario, with a high incidence rate of 5.9/100,000 (Izuierdo et al. 2015). Calculation of a comparative Israeli outbreak-based cost-utility analyses is beyond the scope of this paper as this will involve fewer doses to a far wider age group.

Our $19.3 \%$ neurological sequelae rate was obtained from the follow-up study of 57 MEN B survivors aged 0-14 during 2000-2013 (De Wals et al. 2011), this was higher than 12.5 and $15.8 \%$ rates reported for all serogroups in the USA (Chandran et al. 2011), and in a recent global meta-analysis (Brouwer et al. 2013), respectively.

Our baseline estimate of the CUR could be biased upwards (i.e.: making the vaccine appear to be less favourable economically) due to lack of data on:

(i) possible sequelae, such as visual defects (Sridhar et al. 2015; Chandran et al. 2011; Edmond et al. 2010), behavioural problems (Wright et al. 2013; Edmond et al. 2010], psychological disorders (Wright et al. 2013; Viner et al. 2012).

(ii) payments for resources such as for personal social services, home and environmental adaptations (Wright et al. 2013).

(iii) potential DALY losses due to care burden of spouses or parents of a person handicapped by sequelae.

(iv) work absences in adulthood arising from hearing disorders, seizures, amputations or renal disease.

An additional cause of upward bias is the fact that there has been a downward trend in reported MEN B cases since 2011, and so our incidence estimates, which were based on the 2000-2013 average, may have overestimated the impact of the vaccination.

Evidence for herd immunity was inferred from a French outbreak that suggested meningococcal OMV-based vaccines reduce meningococcal carriage in children aged 1-7 and may hence confer herd immunity (Delbos et al. 2013). A meta-analysis reported that meningococcal carriage increased with age till 19 years and then declined (Christensen et al. 2010). However, while this analysis was carried out in European countries (where disease caused by serogroups $\mathrm{B}$ and $\mathrm{C}$ dominate), no separate analysis was presented for serogroup B on its own. Weaker evidence of herd immunity comes only from MEN C conjugate vaccine studies (Trotter and Edmunds 2006; Ramsay et al. 2003).

A study of English University students reported that MENB vaccines reduced meningococcal carriage rates during the year after vaccination and therefore might affect transmission when widely implemented (Read et al. 2014). The UK guidelines stated that impact on acquisition of carriage would have a limited impact on the cost-effectiveness of an infant programme, since it is driven by direct individual protection rather than by herd immunity (JCVI 2014). As MENB vaccine has only been used at a population level since September 2015, it is not yet known if it will confer herd immunity (Meningococcal B pilot Project Task Group 2014). Therefore, we conservatively assumed, in our baseline analysis, the absence of herd protection. If this assumption is erroneous, then our baseline CUR will be upwardly biased (Trotter and Edmunds 2006).

Our CUR is slightly underestimated (i.e. making the vaccine appear to be more favourable economically) because we were unable to determine the few cases where sequelae were not mutually exclusive hence slightly overestimating averted DALYs. The CUR will also be downwardly biased if there is serogroup replacement as a result of the vaccination. However, no evidence exists as to serogroup replacement four years after the introduction of serogroup C vaccinations in the UK (Trotter et al. 2004). The vaccines true effectiveness will only be revealed through post-licensure surveillance (Gorringe and Pajon 2012). A further cause of downward bias could be that if the secular decline in IMD which was observed over the past 30 years (Israel Center for Disease Control 2012) continues, then the actual number of cases prevented by vaccination would be overestimated.

The spreadsheet model that we used could be simply and quickly adapted (by changing incidence, CFR, DEMOGRAPHIC and cost-parameters) by middle income or developing countries, especially in the meningitis belt of Africa, to evaluate the cost per QALY of introducing childhood vaccinations against MEN B. Such information will be useful for potential funding agencies such as governments or the Global Vaccine Initiative.

In September 2014, the advisory committee on infectious disease and vaccines of the Ministry of Health decided not to recommend the adoption of the new MEN B vaccine into the routine childhood vaccination programme. This was primarily because the extent of vaccine strain coverage is currently undetermined in Israel but should hopefully be available in the near future. It was also noted (on the basis of the results of this paper) that the vaccination at current price levels is unlikely to be cost-effective (even if strain coverage is very high). Vaccination will be 
cost-effective only in high incidence regions or if there will be a substantial decrease in prices.

\section{Compliance with ethical standards}

Funding This study did not receive any funds and was carried out by salaried staff members of their respective institutions.

Ethical approval This article does not contain any studies with human participants or animals performed by any of the authors.

\section{References}

Arnold R, Galloway Y, McNicholas A, O'Hallahan J (2011) Effectiveness of a vaccination program for an epidemic of meningococcal B in New Zealand. Vaccine 29:7100-7106

Ben-Shimol S, Dagan R, Schonmann Y et al (2012) Dynamics of childhood invasive meningococcal disease in Israel during a 22-year period (1989-2010). Infection 41:791-798. doi:10.1007/ s15010-013-0439-6

Bettinger JA, Scheifele DW, Le Saux N, Halperin SA, Vaudry W, Tsang R, For the members of the Canadian immunization Monitoring Program Active (IMPACT) (2013) The disease burden of invasive meningococcal serogroup $\mathrm{B}$ disease in Canada. Pediatr Infect Dis J 32:e20-e25. doi:10.1097/INF. 0b013e3182706b89

Brouwer MC, McIntyre P, Prasad K, van de Beek D (2013) Corticosteroids for acute bacterial meningitis. Cochrane Database Syst Rev 6:CD004405. doi:10.1002/14651858.CD004405. pub4. Accessed 21 Jan 2015

Buysse CMP, Raat H, Hazelet JA et al (2008) Long-term health status in childhood survivors of meningococcal septic shock. Arch Pediatr Adolesc Med 162:1036-1041

Buysse CM, Oranje AP, Zuidema E, Hazalet JA, Hop WCJ, Diepstaten AF, Joosten KFM (2009) Long-term skin scarring and orthopaedic sequelae in survivors of meningococcal septic shock. Arch Dis Child 94:381-386

Buysse CMP, Vermunt LCAC, Raat H, Hazalet JA, Hop WCJ, Utens EMWJ, Joosten KFM (2010) Surviving meningococcal septic shock in childhood: long-term overall outcome and the effect of health-related quality of life. Crit Care 14:R124. doi:10.1186/ cc9087 (Published Online 29 June 2010)

Central Bureau of Statistics (2015) Annual Statistical Abstract 2015. CBS, Jerusalem

Centres for Disease Control and Prevention (2005) Prevention and control of meningococcal disease recommendations of the advisory committee on immunization practices (ACIP). MMWR 54:1-21

Chandran A, Herbert H, Misurski D, Santashan M (2011) Long-term sequelae of childhood bacterial meningitis. Pediatr Infect Dis J 30:3-6

Christensen H, May M, Bowen L, Hickman M, Trotter CL (2010) Meningococcal carriage by age: a systematic review and metaanalysis. Lancet Infect Dis 10:853-861

Christensen H, Hickman M, Edmunds WJ, Trotter CL (2013) Introducing vaccination against serogroup B meningococcal disease: an economic and mathematical modelling study of potential impact. Vaccine 31:2638-2646

Christensen H, Trotter CL, Hickman M, Edmunds WJ (2014) Reevaluating cost effectiveness of universal meningitis vaccination (Bexero) in England: modelling study. BMJ 349:g5725

De Wals P, Deceuninck G, Lefebvre B, Boulianne N, De Serres G (2011) Effectiveness of serogroup $C$ meningococcal conjugate vaccine: a 7-year follow-up in Quebec, Canada. Pediatr Infect Dis J 30(7):566-569. doi:10.1097/INF.0b013e31820e8638
Delbos V, Lemée L, Bénichou J et al (2013) Impact of MenBvac, an outer membrane vesicle (OMV) vaccine, on the meningococcal carriage. Vaccine 31:4416-4420

Dellicour S, Greenwood B (2007) Systematic review: impact of meningococcal vaccination on pharyngeal carriage of meningococci. Trop Med Int Health 12:1409-1421

Edmond K, Clark A, Korczak VS, Sanderson C, Griffiths UK, Rudan I (2010) Global and regional risk of disabling sequelae from bacterial meningitis: a systematic review and meta-analysis. Lancet Infect Dis 10:317-332

Edwards MS, Baker CJ (1981) Complications and sequelae of meningococcal infections in children. J Pediat 99(4):540-545

Erickson L, De Wals P (1998) Complications and sequelae of meningococcal disease in Quebec, Canada, 1990-1994. Clin Infect Dis 26:1159-1164

FDA News Release (2014) First vaccine approved by FDA to prevent serogroup B Meningococcal disease. http://www.fda.gov/ NewsEvents/Newsroom/PressAnnouncements/ucm420998.html. Accessed 1 Jan 2015

Frosi G, Biolchi A, Sapio ML et al (2013) Bactericidal antibody against a representative epidemiological meningococcal serogroup B panel confirms that MATS underestimates 4CMenB vaccine strain coverage. Vaccine 31:4968-4974

Gill C (2013) Novel assessment of a novel meningitis B vaccine. Lancet Infect Dis 5:381-382. doi:10.1016/S14733099(13)70037-9 (Epub 2013 Feb 13)

Ginsberg GM, Kark J, Einav S (2015) Cost utility analysis of cardiac resuscitation services in Jerusalem. Resuscitation 86:54-61

Gorringe AR, Pajon R (2012) Bexsero: a multicomponent vaccine for prevention of meningococcal disease. Hum Vaccines Immunother 8:174-183

Gossger N, Snape MD, Yu LM, European MenB Vaccine Study Group et al (2012) Immunogenicity and tolerability of recombinant serogroup B meningococcal vaccine administered with or without routine infant vaccinations according to different immunization schedules: a randomized controlled trial. JAMA 307(6):573-582

Hakin B, Cosford P (2015) Introduction of Men B immunisation for infants. Public Health England, NHS Gateway Number: 03517. https://www.gov.uk/government/uploads/system/uploads/ attachment_data/file/437906/150622_MenB_bipartite_letter.pdf

Healy CM, Butler KM, Smith EO et al (2002) Influence of serogroup on the presentation, course, and outcome of invasive meningococcal disease in children in the Republic of Ireland, 1995-2000. Clin Infect Dis 34:1323-1330

Holst J, Feiring B, Fuglesang JE, Høiby EA, Nøkleby H, Aaberge IS, Rosenqvist E (2003) Serum bactericidal activity correlates with the vaccine efficacy of outer membrane vesicle vaccines against Neisseria meningitis serogroup B disease. Vaccine 21:734-737

Huels J, Wassil J, Bonefeld L, Watson P (2013) Sensitivity analysis of a model used to predict the cost effectiveness of bexero for immunisation against meningococcal disease in the United Kingdom. In: Poster presented at meningitis and septacemia in children and adults, 5-6 November 2013, London

Israel Center for Disease Control (2012) Notifiable infectious diseases in Israel, 60 years of surveillance 1951-2010. Publication No. 342

Izuierdo G, Torres JP, Santolya ME, Valenzuela MT, Vega J, Chomali M (2015) Cost-effectiveness analysis of a multicomponent meningococcal serogroup B (4CMeb B) vaccine in epidemic situation in a middle-income country. Hum Vaccine Immunother 11:875-883. doi:10.1080/21645515.2015.1010885

Jacobs P, Noseworthy TW (1990) National estimates of intensive care utilization and costs. Crit Care Med 18:1282-1286

JCVI (2014) JCVI position statement on use of bexsero meningococcal B vaccine in the UK. 2014. https://www.gov.uk/ 
government/publications/meningococcal-b-vaccine-jcvi-positionstatement. Accessed 21 Aug 2014

Lozano R, Naghavi M, Foreman K et al (2012) Global and regional mortality from 235 Causes of Death for 20 age groups in 1990 and 2010: a systematic analysis for the Global Burden of Disease Study 2010. Lancet 380:2095-2128

McNicholas A, Galloway Y, Stehr-Green P et al (2007) Postmarketing safety monitoring of a new group B meningococcal vaccine in New Zealand, 2004-2006. Hum Vaccines 3:196-204

Meningococcal B Pilot Project Task Group (2014). The recommended use of the multicomponent meningococcal $B$ (4CMENB) vaccine in Canada: common guidance statement. Ottawa

Ministry of Health (2012) Ambulatory and hospitalization price data July 1st 2012. http://www.health.gov.il/subjects/finance/taarifon/ pages/pricelist.aspx. Accessed 11 July 2013

Novartis Media release (2012) Novartis receives positive CHMP opinion for Bexsero ${ }^{\circledR}$, a groundbreaking vaccine to help prevent devastating MenB infections, the leading cause of meningitis in Europe. http://www.novartis.com/newsroom/media-releases/en/ 2012/1658747.shtml. Accessed 15 May 2013

Pouwels KB, Hak E, van der Ende A, Christenden H, van der Dobbelstein GP, Postma MJ (2013) Cost-effectiveness of vaccination against meningococcal B among Dutch infants. Hum Vaccines Immunother 9:1-10

Ramsay ME, Andrews NJ, Trotter CL, Kaczmarski EB, Miller E (2003) Herd immunity from meningococcal serogroup C conjugate vaccination in England: database analysis. BMJ 326:365-366

Read RC, Baxter D, Chadwick DRI et al (2014) Effect of a quadrivalent meningococcal ACWY glycoconjugate or a serogroup B meningococcal vaccine on meningococcal carriage: an observer-blind, phase 3 randomised clinical trial. Lancet 384:2123-2131

Scholten RJ, Bijlmer HA, Valkenburg HA, Dankert J (1994) Patient and strain characteristics in relation to the outcome of meningococcal disease: a multivariate analysis. Epidemiol Infect 112:115-124

Scott RD, Meltzer MI, Erikson LJ, DeWals P, Rosenstein NE (2002) Vaccinating first-year college students living in dormitories for meningococcal disease. Am J Prev Med 23:98-105

Slack R, Hawkins KC, Gilhooley L, Addison GM, Lewis MA, Webb NJA (2005) Long-term outcome of meningococcal sepsisassociated acute renal failure. Pediatr Crit Care Med 6:477-479

Sridhar S, Greenwood B, Head C et al (2015) Global incidence of serogroup $\mathrm{B}$ invasive meningococcal disease: a systematic review. Lancet Infect Dis 15:1334-1346. doi:10.1016/S14733099(15)00217-0 (Epub 2015 Oct 6)

Stein-Zamir C, Abramson N, Zentner G, Sloob H, Valinsky L, Block $C$ (2008) Invasive meningococcal disease in children in Jerusalem. Epidemiol Infect 136:82-89

Stein-Zamir C, Shoob H, Sokolov I, Kunbar A, Abramson N, Zimmerman D (2014) The clinical features and long-term sequelae of invasive meningococcal disease in children. Pediatr Infect Dis J 33:777-779

Tirani M, Meregaglia M, Melegaro A (2015) Health and economic outcomes of introducing the new Men B vaccine (bexero) into the Italian routine infant immunisation programme. PLoS One 10:e0123383. doi:10.1371/journal.pone.0123383

Trotter CL, Edmunds WJ (2006) Reassessing the cost-effectiveness of meningococcal serogroup $\mathrm{C}$ conjugate (MCC) vaccines using a transmission dynamic model. Med Decis Mak 26:38-47

Trotter CL, Andrews NJ, Zaczmarski EB, Miller E, Ramsay ME (2004) Effectiveness of meningococcal serogroup $\mathrm{C}$ conjugate vaccine 4 years after introduction. Lancet 364:365-367

Tu HA, Deeks SL, Morris SK et al (2014) Economic evaluation of meningococcal serogroup B childhood vaccination in Ontario, Canada. Vaccine 32:5436-5446

Vesikari T, Eposito S, Ypma E et al (2013) Immunogenicity and safety of an investigational multicomponent, recombinant, meningococcal serogroup B vaccine (4CMEN B) administered concomitantly with routine infant and child vaccinations: results of two randomized trials. Lancet 381:825-35 (Erratum in: Lancet (2013) 381: 804)

Viner RE, Booy R, Johnson H et al (2012) Outcomes of invasive meningococcal serogroup $\mathrm{B}$ disease in children and adolescents (MOSAIC): a case-control study. Lancet Neurol 11:774-783

WHO Commission on MacroEconomics and Health (2001) Macroeconomics and health: investing in health for economic development. Report of the Commission on Macroeconomics and Health. World Health Organization, Geneva, p 2001

Wise J (2014) News: meningitis B vaccine to be introduced in UK after $\mathrm{U}$ turn on its cost effectiveness. BMJ 348:g2327. doi:10. 1136/bmj.g2327

World Health Organization (1996) Murray CJL, Lopez AD (eds) The global burden of disease: a comprehensive assessment of mortality and disability from diseases, injuries and risk factors in 1990 and projected to 2020. WHO, USA

Wright C, Wordsworth R, Glennie L (2013) Counting the cost of meningococcal disease: scenarios of severe meningitis and septicemia. Paediatr Drugs 15:49-58 